\title{
A Mobile-based Face Verification System
}

\author{
Zongying Ou, Tieming Su, Fan Ou, Jianxin Zhang, Dianting Liu \\ $C A D 、 C G \&$ Network Lab. Dalian University of Technology, China \\ E-mailouzyg@dlut.edu.cn
}

\begin{abstract}
Intelligent mobile phones equipped with camera are very popular in human daily lives now. Face verification running on mobile phone provides not only a tool for protection of owner's authority but also an approach for verification from a distance. The combination of biometrics and telecommunication technologies possesses broad application potentials in information exchanges. However, there are also some critical issues needed solving. Usually the processing power and memory size of a mobile phone system are limited, and the acquisition images are suffered from the illumination changes. Based on the invariant property of the ratio of illumination at a pixel to its surrounding neighbor illuminations, an illumination normalization technique was analyzed and adapted to the mobile system. A dedicated scheme composed with special wavelets called Gab features was proposed for representation of human faces. The number of Gabor nodes was effectively reduced by an optimization processing. A mobile based face verification system was developed and embedded in Lenovo ET980 mobile phone. The experiments testing on FERET fafb dataset show that the performance of this system is quite well.
\end{abstract}

\section{Introduction}

Compared with traditional identification security technologies, biometrics provides more superior performances. Based on the statistics and estimation by Inter-national Biometric Group, the worldwide biometric market figures in 2005 are about $1,540,000,000 \mathrm{USD}$, and will be growing up to $5,700,000,000$ USD in 2010 [1].

Face identification is the most convenient and userfriendly biometric technology. As an effective and portable telecommunication tool, mobile phones equipped with cameras are getting popular in human daily lives; implementation of face verification on mobile phones not only lets mobile phone owners keep their ownership more security and safety, but also provides a simple way in establishing flexible identification tele-networks via mobile communication, which do have broad potentials in the growing market fields.

Research work on face identification has been carried for many years and much progress has been made [2-7]. However, compared with the conventional fixed computer system, usually the processing power of the CPU and the capacity of the main memory in portable mobile systems are limited, and the illumination environments are in uncontrolled conditions, which lead the development of a practical mobile face verification system to be a challenge task. Recently, a mobile-based face verification system was developed by CAD、CG \& Network Lab, Dalian University of Technology. Much attention has been paid to upgrade the processing efficiency and the robustness against the illumination changes in verification processing. The experiments testing on FERET fafb dataset [8] and other dataset show that the system works well.

\section{System Architecture and working flow}

The verification system contains two modules: Model training module and Verification module. The verification module will run on Window CE 5.0 platform, and takes the LENOVO ET980 intelligent mobile phone as hardware platform, which is equipped with CPU Intel XScale PX270, memory 128MB Flash/ 64 MB SDRAM and a 4 million pixel camera. Before putting verification module into implementation the verification model training module should run first to yield the model parameters for verification. The training module will run on a table computer since it involves much digital calculation and machine learning processing.

The working flow is as follows: 


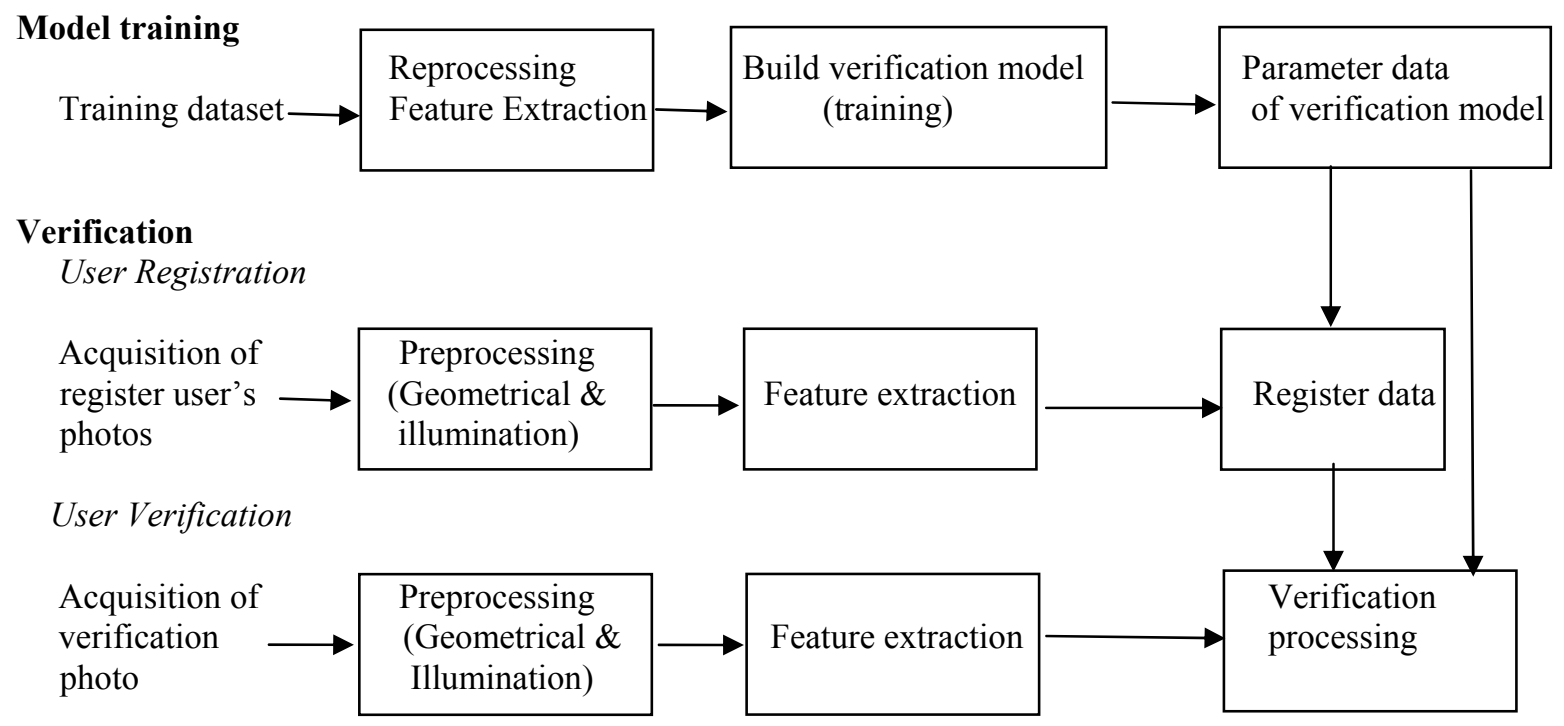

Figure 1. Processing flow chart

The geometrical preprocessing is clipping the detected face and aligning it to a normal scale and position specified with two eye's locations.

The illumination preprocessing is to normalize the varieties of illumination in acquisition photos. This is a critical factor affecting the verification correctness, since working environments for mobile phones are quite different. It has been shown that the differences of face images caused by varying illumination are more significant than the inherent differences between individuals [9]. Illumination normalization is a very important processing in face verification.

An acquisition image $I(x, y)$ can be modeled as a product of the reflectance $\mathrm{R}$ and Illuminant effect $\mathrm{L}$, the fundamental relation can be shown briefly as follows:

$$
I(x, y)=R(x, y) * L(x, y)
$$

where $(x, y)$ are pixel coordinates; $I(x, y)$ is the Intensity of image at pixel $(x, y) ; R(x, y)$ is the reflectance attribution of a face at pixel $(x, y) ; L(x, y)$ is the illuminate effect at pixel $(x, y)$.

The reflectance attribution $R(x, y)$ is the inherent feature of a human face, and the discriminating features for verification should be formed based on these attributions. It is an ill-posed problem to extract the exact $R(x, y)$ from a given image $I(x, y)$ based on relation (1), since the illumination term is unknown. To solve this problem, some approximate assumptions had to be made. The intensity ratio between two pixels is:

$$
\frac{I(x 1, y 1)}{I(x 2, y 2)}=\frac{R(x 1, y 1)}{R(x 2, y 2)} \cdot \frac{L(x 1, y 1)}{L(x 2, y 2)}
$$

Compared with the reflectance changes rapidly across the contour of a face, usually the illumination changes smoothly and slowly. If these two pixels locate nearby and there is no shadow between them, the illumination at these pixel's positions can be assumed as approximately equal. Then the following simplified relation can be established:

$$
\frac{I(x 1, y 1)}{I(x 2, y 2)}=\frac{R(x 1, y 1)}{R(x 2, y 2)}
$$

This relation means that ratio of reflectance can be calculated via ratio of illuminations, which is approximately independent from illumination changes. The ratio of the intensity at a pixel to a specific synthetic intensity, which is the convolution result from the intensities of surrounding pixels, reflects the illumination reflectance feature at this pixel [10]. For calculation convenience we take the logarithmic ratio as normalized illumination, which is calculated as follows:

$\bar{I}(x, y)=\log (I(x, y)-\log (G(x, y) * I(x, y))$

where $\bar{I}(x, y)$ is the normalized illumination; * denotes a convolution operation; $G(x, y)$ is a Gaussian surround function, assuming that the influence of a pixel depends on the distance to the center and decays as Gaussian function description. 


$$
\left.G(x, y)=K \exp \left((\Delta x)^{2}+(\Delta y)^{2}\right) / c\right)
$$

where $\Delta x, \Delta y$ are the distances to the center, $K$ is an adjustable constant, adopted to

$$
\left.\int_{-\infty}^{\infty} K \exp \left((\Delta x)^{2}+(\Delta y)^{2}\right) / c\right)=1
$$

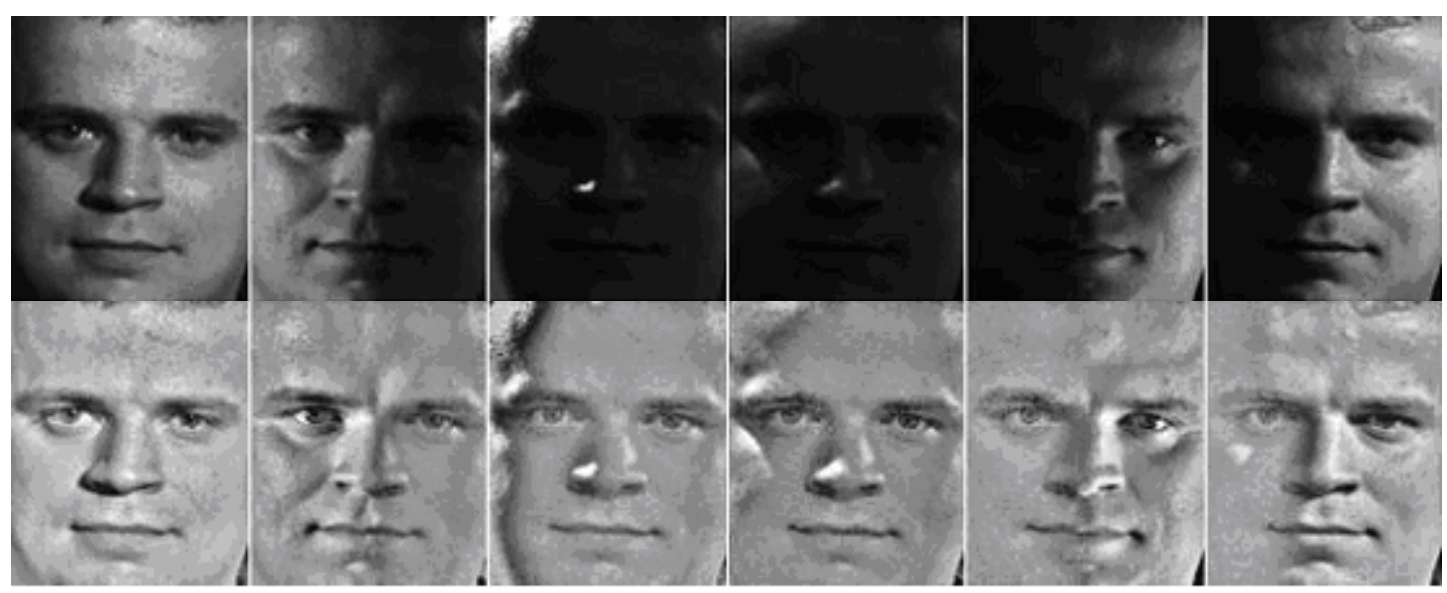

Figure 2. Illumination normalization results on Yale face database B

\section{Discriminating Feature selection and Dimension Reduction}

A 120X100 face image contains 12000 pixel intensity data. The intensity at a pixel is independent from others and is sensitively affected by noise. The pixel intensity based face representation is not a good representation for verification, since it lacks of high level intrinsic connection features. A wavelets based representation call Gabor feature representation was proposed for face image processing and has been successfully applied in face identifications. Gabor filters are defined as follows:

$$
\begin{aligned}
& \psi_{u, v}(\vec{x})=\left(\left\|k_{u, v}\right\|^{2} / \sigma^{2}\right) \exp \left(-\left\|k_{u, v}\right\|^{2}\|x\|^{2} / 2 \sigma^{2}\right) . \\
& \left(\exp \left(i k_{u, v} \cdot x\right)-\exp \left(-\sigma^{2} / 2\right)\right)
\end{aligned}
$$

where $u$ and $v$ define the orientation and scale, and the wave vector $k_{u, v}$ is defined as follows:

$$
\begin{gathered}
k_{u, v}=k_{v} \exp \left(i \phi_{v}\right) \\
k_{v}=k_{\max } / f^{v} \\
\phi_{u}=u \pi / 8
\end{gathered}
$$

where $c$ is a scale parameter, small $c$ possesses stronger power in remedying uneven lightness; however, increasing $c$ magnitude can get more smooth normalization output.

Yale face database B [11] is used for evaluating the effect of illumination normalization, some typical results are shown in Figure 2. where $k_{\max }$ is the maximum frequency and $f$ is the sp acing factor between kernels in the frequency domain.

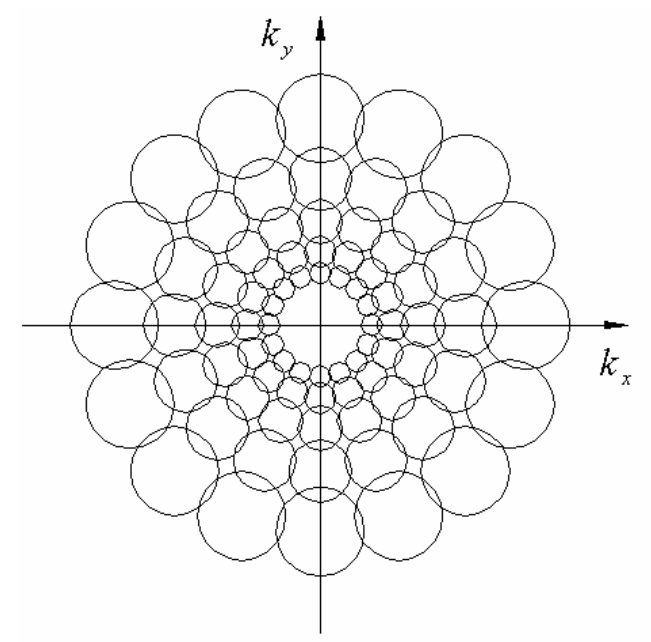

Figure 3. Arrangement diagram of 40 Gabor filters in frequency plane at a node

In face recognition application, we use the Gabor filters with the following parameters: five scales $v \in\{0, \ldots, 4\} \quad ; \quad$ eight orientations $u \in\{0, \ldots, 7\}$; $k_{\text {max }}=\pi / 2 ; f=\sqrt{2} ; \sigma=2 \pi$, there are $5 * 8=40$ Gabor filters at a node and shown as Figure 3.

A $128 \times 100$ face image possesses 12800 pixels. If take every pixel as one node, then the total number of 
Garbor features will be $12800 * 40=512000$, which is a huge number. In view of the active scope of a Gabor feature is a circle area covering its surrounding region, we can distribute the nodes sparsely. Further investigations show that some regions like eyebrow, eye contour and nose do provide higher discriminating information, we can neglect some nodes and only select the important nodes with an optimization processing [12]. An evenly distribution configuration scheme of nodes was shown in Figure 4; based on this scheme an optimization configuration scheme composed of 56 nodes was shown in Figure 5.

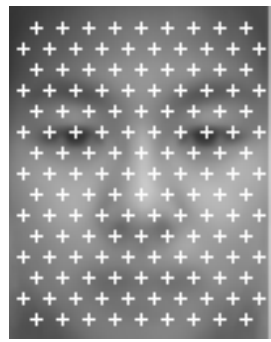

Figure 4. Gabor node configuration with evenly distribution (142 nodes)

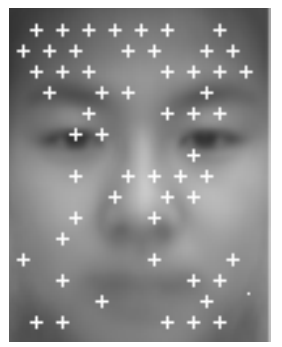

Figure 5. Gabor node configuration with optimal distribution (56 nodes)

\section{Implementation and Experiments}

A prototype mobile face verification system was developed running on Lenovo mobile phone ET980 shown as figure 6-7., the technical specifications are: CPU Intel XScale PX270 $312 \mathrm{MHz}$ Memory 128MB Flash 64 MB SDRAM

Camera 4 million pixels

Screen $240 X 320$ resolutions

Operating system Microsoft Pocket PC 2005 Phone Edition

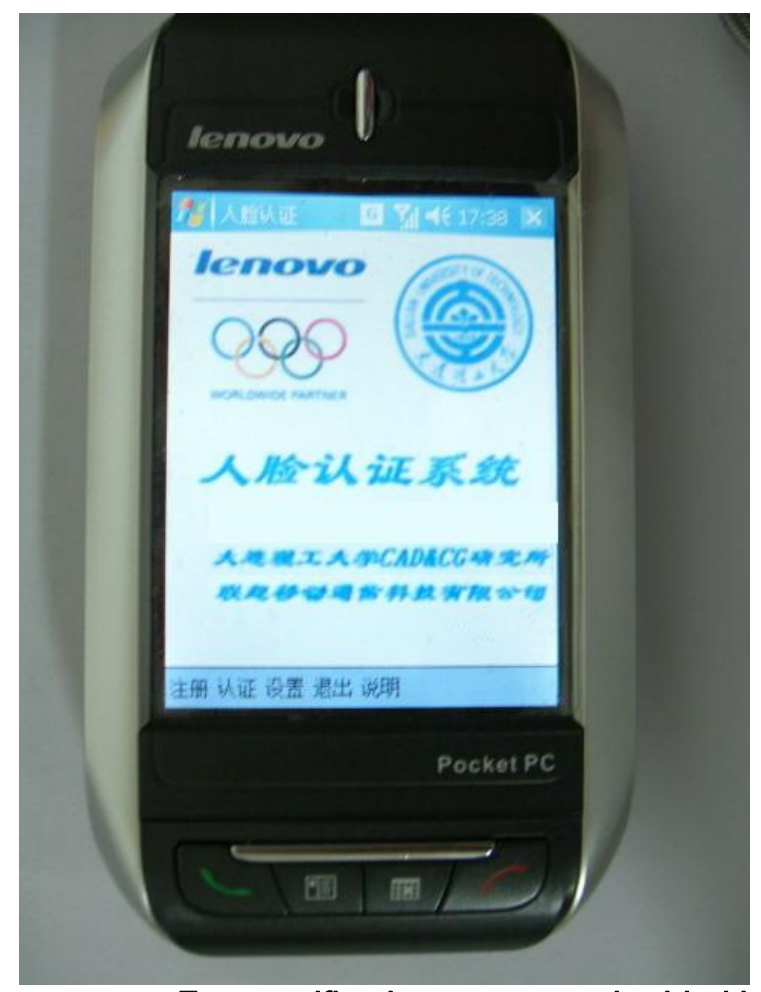

Figure 6. Face verification system embedded in Lenovo ET980 mobile phone

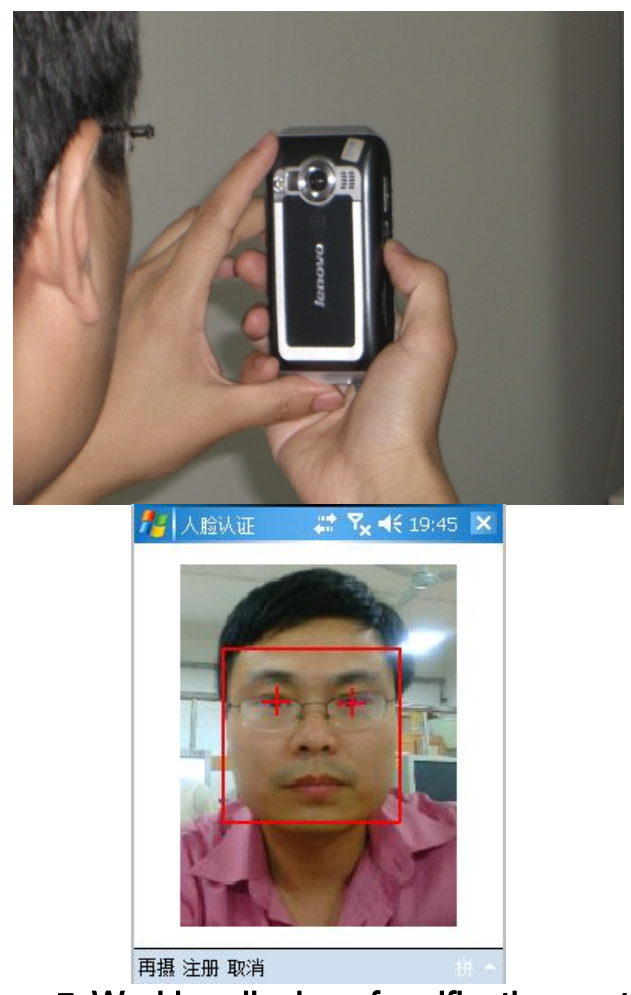

Figure 7. Working display of verification system 
The system was built based on the following techniques: normalization illumination by the ratio of intensity at a pixel to intensities of local surrounding pixels; extraction discriminating Gabor feature with optimized 56 node scheme configuration; classifying with linear discriminant analysis (LDA) algorithm; all the floating point calculations are implemented by integer calculation. For comparison, the public FERET fafb dataset was used for testing. The testing results are:

ERR (Equal False Accept Rate and

False Reject Rate)

$1.51 \%$

FAR100 (False Accept Rate at False

Reject Rate equal to $1 \%$ )

$2.12 \%$

Far1000 (False Accept Rate at False

Reject Rate equal to $0.1 \%$ )

Processing time

$6.91 \%$

$<1$ second

\section{Summary}

Mobile phones with embedded cameras are popular telecommunication tools. Face verification technology embedded on mobile phone not only provides a tool for protecting the owner's authority .but also provides convenient approaches for implementation of televerification which is very important in authorized human contact.

Compared with fixed computer system, mobile phones are usually equipped with weaker performance processor and smaller memory while works on varieties of uncontrolled environments. Illumination normali-zeation and simplifying the processing of extracting features and classifying yet still keeping high precision are two key issues in developing practical mobile based verification system.

Using the ratio of the intensity at a pixel to the intensities of its surrounding pixels as normalized Illumination can represent the intrinsic reflectance of face, which keeps approximately invariant while illumination changes. Optimization of the configuration of Gabor nodes can reduce the calculation of Gabor feature significantly.

A mobile based verification system developed by CAD、CG \& Network Lab of Dalian University of Technology has been implemented on Lenovo E980 mobile phone, the testing results show that the ERR (Equal Error Rate) is about $2 \%$ on pubic FERET fafb dataset while the processing time takes less than 1 second, the performance is quite well.

\section{Acknowledgements}

The research work presented in this paper was supported by research fund DUT-SIA2006.

\section{References}

[1] http://www.biometricgroup.com/reports/public/market_re port.html.

[2] W. Zhao, R. Chellappa, A. Rosenfeld, P.J. Phillips, "Face Recognition: A Literature Survey", ACM Computing Surveys, 2003, pp. 399-458.

[3] M. Turk and A. Pentland, "Eigenface for recognition", Journal of Cognitive Neuoscience, 1991(3):71-86.

[4] P. Belhumeur, J. Hespanha, and D. Kriegman, "Eigenfaces vs. Fisherfaces: Recognition using class specific linear projection", IEEE Transactions on Pattern Analysis and Machine Intelligence, 1997, 19(7):711-720.

[5] C.J. Liu, and H. Wechsler, "Enhanced Fisher Linear Discriminant Models for Face Recognition", In: Proceedings of International Conference Pattern Recognition, Austria, Brisbane, 1998.

[6] L. Wiskott, J.M. Fellous, N. Kruger, et al., "Face recognition by elastic bunch graph matching", IEEE Transaction on Pattern Analysis and Machine Intelligence, 1997,19(7):775-779.

[7] P.J. Phillips, W.T. Scruggs, A.J. O'Toole, P.J. Flynn, K.W. Bowyer, C.L. Schott, and M. Sharpe, "FRVT 2006 and ICE 2006 Large-Scale Results", Technical report, National Institute of Standards and Technology, NISTIR 7408, March 2007. http://iris.nist.gov/ice.

[8] http://www.nist.gov/humanid/colorferet/home.html

[9]Y. Adini, Y. Moses, and S, Ullman, "Face Recognition: The problem of compensating for changes in Illumination direction", IEEE Transaction on Pattern Analysis and Machine Intelligence, 1997, 19(7), pp. 721-732.

[10] D.J. Johson,Z. Rahman, and G.A. Woodell, “ Properties and performance of a center/surround retinix", IEEE Transaction on Image Processing, 1997(6), pp. 451-462.

[11] A.S. Georghiades, and P.N. Belhumeur, and D.J. Kriegman, "From Few to Many: Illumination Cone Models for Face Recognition under Variable Lighting and Pose", IEEE Transaction on Pattern Analysis and Machine Intelligence, 2001, 23(6), pp.643-660.

[12] F. Ou, C. Liu, Y. Li, Z. Ou, “ Evaluation and seletion of discriminating Gabor features for face recognition", Proceedings of 2007 IEEE Workshop on Automatic Identification Advanced Technologies, 2007, pp. 93-98. 